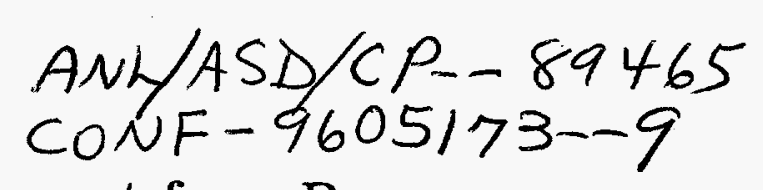

\title{
Positron Beam Position Measurement for a Beam Containing Both Positrons and Electrons*
}

\author{
N. S. Sereno, R. Fuja \\ Advanced Photon Source, Argonne National Laboratory, \\ 9700 South Cass Avenue, Argonne, IL 60439
}

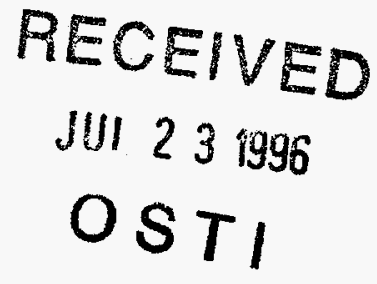

\section{Abstract}

Positron beam position measurement for the Advanced Photon Source (APS) linac beam is affected by the presence of electrons that are also captured and accelerated along with the positrons. This paper presents a method of measuring positron position in a beam consisting of alternating bunches of positrons and electrons. The method is based on Fourier analysis of a stripline signal at the bunching and first harmonic frequenciess. In the presence of a mixed species beam, a certain linear combination of bunching and first harmonic signals depends only on the position and charge of one specie of particle. A formula is derived for the stripline signal at all harmonics of the bunching frequency and is used to compute expected signal power at the bunching and first harmonic frequencies for typical electron and positron bunch charges. The stripline is calibrated by measuring the signal power content at the bunching and first harmonic frequencies for a single species beam. A circuit is presented that will be used with an APS positron linac stripline beam position monitor to detect the bunching and first harmonic signals for a beam of positrons and electrons.
\end{abstract}

\section{INTRODUCTION}

The $7-\mathrm{GeV}$ Advanced Photon Source (APS) is a third-generation light source optimized to produce insertion-device $\mathrm{x}$-ray radiation for materials science research. The APS linear accelerator is used to accelerate a positron beam to $450 \mathrm{MeV}$ and deliver it to a positron accumulator ring (PAR). The beam is accumulated and damped in the PAR, transported to the booster synchrotron and accelerated to $7 \mathrm{GeV}$, and finally injected into a storage ring containing insertion device undulators. The large emittance of the positron beam in the linac requires accurate positron beam position measurement to minimize beam loss during transport to the PAR. In addition, positron position measurement is essential so that response matrix measurements can be performed to verify linac optics for various configurations used to produce and transport positrons.

Figure 1 shows the layout of the APS linac. The top part of the figure (the electron linac) shows the $100-\mathrm{keV}$ gun electron source, accelerating waveguides, and magnets up to the positron production target. The bottom part of the figure (the positron linac) shows the linac S-band (2856 MHz) accelerating waveguides and magnets after the target. The electron beam on the

\footnotetext{
*Work supported by U.S. Department of Energy, Office of Basic Energy Sciences, under Contract No. W-31-109-E.NG-38
}

The submitted manuscript has been authored by a contractor of the U.S. Government under contract No. W-31-109-ENG-38. Accordingly, the U.S. Government retains a nonexclusive, rovalty-free license to publish or reproduce the published form of this contribution, or allow others to do so, for DISTRIBUTION OF THIS DOCUMENT IS UNLIMITED
U.S. Government purposes. 


\section{DISCLAIMER}

Portions of this document may be illegible in electronic image products. Images are produced from the best available original document. 

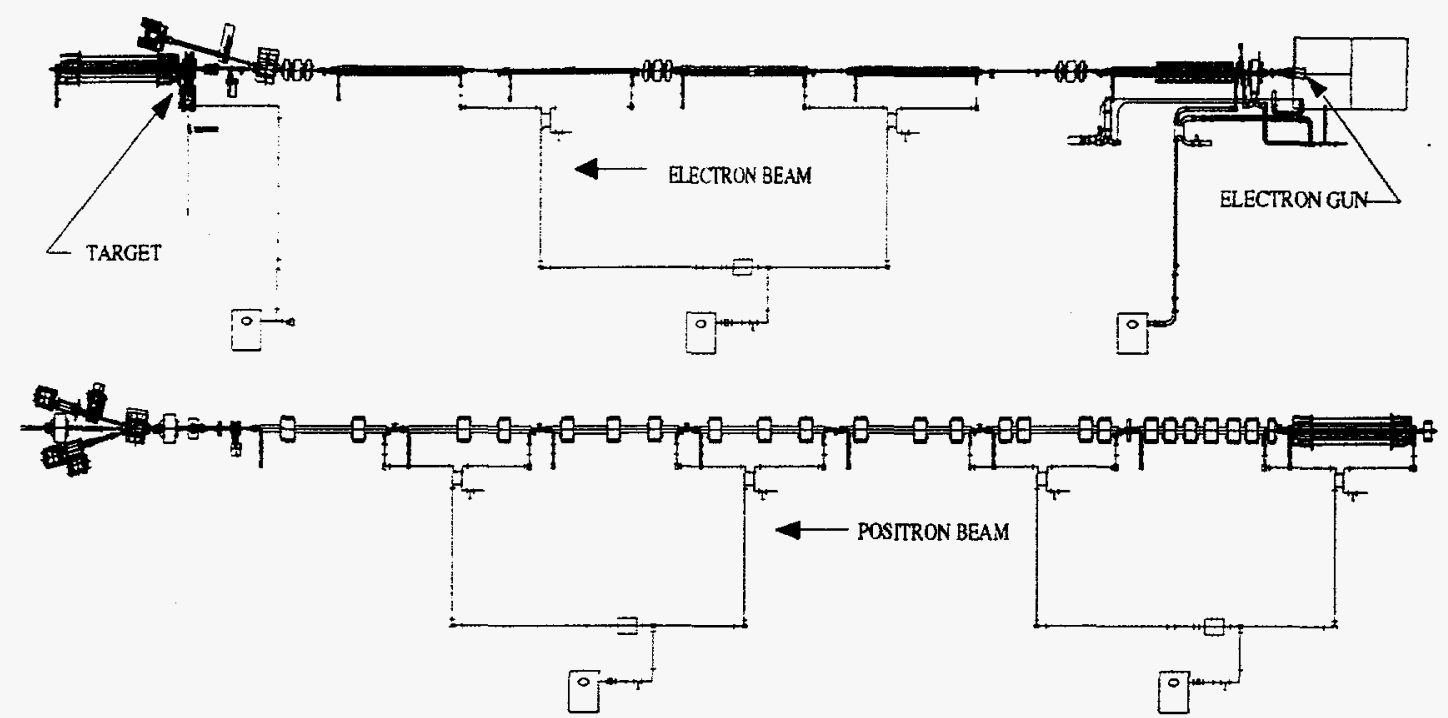

Figure 1. Layout of the APS linear accelerator showing the gun, accelerating waveguides, magnets, and target. The top part of the figure shows the linac layout from the $100-\mathrm{kV}$ gun to the target. The bottom part of the figure shows the linac layout from the target to the end. Stripline BPMs are located between the accelerating waveguide sections.

target is $200 \mathrm{MeV}$ and produces both positrons and electrons through the bremsstrahlung process. Stripline beam position monitors (BPMs) are located throughout the linac between waveguides to monitor beam position. The difficulty of determining positron beam position downstream from the target is that both species of particle are captured and accelerated by the rf.

\section{STRUCTURE OF THE POSITRON LINAC BEAM IN THE TIME DOMAIN}

The parameters that define the linac beam (1) are driven by the requirement that the PAR be completely filled with $6.6 \mathrm{nC}$ of positrons in 0.5 seconds. The electron beam striking the target is a macropulse $30 \mathrm{~ns}$ long repeated at a $60-\mathrm{Hz}$ rate with a peak current of 1.7 amperes. Each macropulse is made up of 86 micropulses repeated at a $2856-\mathrm{MHz}$ rate. After the target, the beam consists of both positrons and electrons that are captured by the rf fields of the first accelerating waveguide. The positron and electron micropulses are separated by half an rf wavelength within each macropulse, forming an alternating microbunch charge pattern. The design peak current for the positron part of the macropulse is $8 \mathrm{~mA}$. The electron current for each macropulse is typically around $6 \mathrm{~mA}$ but can be larger or smaller for different linac configurations.

Based on the comments in the previous paragraph, the current in each macropulse as a function of time can be written as

$$
I(t)=\sum_{n=-N}^{N} Q_{p} f_{p}(t-n \tau)-Q_{e} f_{e}\left(t-\left(n+\frac{1}{2}\right) \tau\right) .
$$


where $Q_{p}$ and $Q_{e}$ are the absolute values of the positron and electron charges in each micropulse (assumed constant as a function of the index $n$ ), $f_{p}(t)$ and $f_{e}(t)$ are the positron and electron normalized distributions, and $\tau$ is the temporal bunch spacing. The micropulse distribution for each particle is assumed different to take into account the fact that the bunching process is different for electrons and positrons. For the APS linac beam, there are $(2 N+1)=86$ electron and positron micropulses per 30 -ns macropulse and the temporal bunch spacing is determined by the $\mathrm{S}$-band rf frequency $(2856 \mathrm{MHz})$.

\section{BPM STRIPLINE SIGNALS GENERATED BY THE POSITRON LINAC BEAM IN THE TIME DOMAIN}

The APS linac BPMs consist of striplines designed to be a quarter wavelength long at the rf frequency of $2856 \mathrm{MHz}$. The stripline output is a bipolar voltage pulse for each beam micropulse that passes along its length (2). In the time domain, the stripline output voltage is written as

$$
V(t)=Z\left\{I_{w}(t)-I_{w}(t-2 \alpha \tau)\right\}
$$

where $\alpha$ is the stripline length in units of wavelength at $2856 \mathrm{MHz}, Z$ is the stripline impedance $(50 \Omega)$, and $I_{w}(t)$ is the wall current induced in the stripline electrode. The linac stripline electrical length is actually measured to be $0.21 \lambda$. The wall current is proportional to the beam current given by Eq. (1) where the proportionality constant is a function of transverse position and stripline geometry (3).

In the analysis that follows, the position dependence in Eq. (2) is suppressed and the wall current is simply written as the beam current in addition to some geometry factors. This is justified because the analysis presented here is with respect to the time and frequency domains, and the position of the particles in the beam is considered constant over the 30 ns it takes to measure the beam pulses. In the rest of the analysis presented here, one should keep in mind that the beam current terms in the equations contain implicitly the transverse position information being sought.

\section{BPM STRIPLINE SIGNALS GENERATED. BY THE POSITRON LINAC BEAM IN THE FREQUENCY DOMAIN}

The bipolar stripline voltage pulses given by Eq. (2) are now analyzed in the frequency domain. Taking the Fourier transform of Eq. (2) yields

$$
\begin{aligned}
V(\omega) & =\frac{Z \phi}{4 \pi}\left(1-e^{-2 i \alpha \omega \tau}\right)\left(Q_{p} F_{p}(\omega)-e^{-\frac{i \omega \tau}{2}} Q_{e} F_{e}(\omega)\right) k_{N}(\omega) \\
k_{N}(\omega) & =\frac{\sin \left\{(2 N+1) \frac{\omega \tau}{2}\right\}}{\sin \left(\frac{\omega \tau}{2}\right)}
\end{aligned}
$$

where $F_{p}(\omega)$ and $F_{e}(\omega)$ are the positron and electron bunch spectra and $\phi$ (typically on the order of 1 radian) is the angle subtended by the stripline 
electrode. The bunch spectra roll off at frequencies on the order of the reciprocal positron and electron rms microbunch length. For the APS linac, the rms bunch length is on the order of 5 ps resulting in rolloff of the electron and positron bunch spectra at frequencies on the order of $200 \mathrm{GHz}$. The quantity $k_{N}(\omega)$ is a strongly peaked function of frequency at the bunching frequency harmonics. A good measure of the width of each peak is given by the difference in frequency between the first zeros of the numerator of Eq. (4) on either side of the peak. This bandwidth is given by

$$
\delta \omega=\frac{2 \omega_{0}}{2 N+1},
$$

where $\omega_{0}=1 / \tau$ is the rf bunching frequency (2856 MHz). Using Eq. (5), the APS linac macropulse length of $30 \mathrm{~ns}$ ( 86 micropulses of electrons and positrons) means that the stripline spectrum has appreciable power content over a bandwidth of "66.4 $\mathrm{MHz}$ around each harmonic of the rf frequency.

The stripline power spectrum is now computed for each bunching frequency harmonic for the bandwidth given by Eq. (4). The power spectrum is defined by (4)

$$
P\left(n \omega_{0}\right)=\frac{1}{\Delta} \int_{n \omega_{0}-\frac{\delta \omega}{2}}^{n \omega_{0}+\frac{\delta \omega}{2}} \frac{|V(\omega)|^{2}}{Z} d \omega,
$$

where $\Delta=30$ ns is the width of the macropulse. Evaluation of this integral is greatly simplified by the fact that the amplitude spectrum is strongly peaked so that over the bandwidth given by Eq. (5) the bunch spectra are constant. The only factor that remains under the integral after this simplification is the function $k_{N}(\omega)^{2}$. Performing the integration results in

$$
\begin{aligned}
P\left(n \omega_{0}\right) & =\left(\frac{Z \phi^{2} \Delta}{8 \pi^{2} \tau}\right)\left(\frac{I_{N}}{(2 N+1)^{2}}\right) \sin ^{2}(2 \pi n \alpha) F\left(n \omega_{\circ}\right)^{2}\left(I_{p}-(-1)^{n} I_{e}\right)^{2} \\
I_{N} & =\frac{\tau}{2} \int_{n \omega_{0}-\frac{\delta \omega}{2}}^{n \omega_{0}+\frac{\delta \omega}{2}} k_{N}^{2}(\omega) d \omega=243.9
\end{aligned}
$$

where

$$
Q_{p}=\frac{I_{p} \Delta}{2 N+1}
$$

and

$$
Q_{e}=\frac{I_{e} \Delta}{2 N+1}
$$

are the average positron and electron current in the 30-ns macropulse. The electron and positron bunch spectrum $F\left(n \omega_{0}\right)$ is taken to be the same for both positrons and electrons because for short bunch lengths far from rolloff, these factors are equal to unity for any arbitrary bunch distribution. For the APS linac BPM application considered in this paper the fundamental and first harmonic stripline signals need to be detected. Using Eq. (7), the fundamental 
$(n=1)$ and first harmonic $(n=2)$ have a power content of $1.2 \mathrm{dBm}(257 \mathrm{mV}$ into $50 \Omega$ ) and $-22 \mathrm{dBm}(18 \mathrm{mV}$ into $50 \Omega$ ) for typical positron and electron average currents of $8 \mathrm{~mA}$ and $6 \mathrm{~mA}$, respectively.

For the APS linac striplines, the small first harmonic signal is unavoidable since the stripline length is so close to a quarter wavelength. For position detection of a mixed species beam, an improved stripline configuration would be to construct each stripline to be equal to one-eighth wavelength. This particular stripline length would make the geometry factor in Eq. (7) unity for the first harmonic signal and reduce the fundamental by $3 \mathrm{~dB}$. This is not a problem because the fundamental is proportional to the algebraic sum of the average currents for each particle species in the beam and hence is always much stronger than the first harmonic signal.

The voltage amplitude as a function of harmonic number is related to the power according to Eq. (10) by

$$
V\left(n \omega_{0} \dot{j} \propto \sqrt{P\left(n \omega_{0}\right)} \propto \sin (2 \pi n \alpha)\left(I_{p}-(-1)^{n} I_{e}\right)\right.
$$

where we consider $F\left(n \omega_{0}\right)=1$ for short microbunches and frequencies far from rolloff. This equation shows that for each stripline, if a linear combination of fundamental and first harmonic signals is made, the current and implicit position dependence of one or the other species of particle can be eliminated. Using Eq. (11), the linear combination of fundamental and first harmonic voltages becomes

$$
\begin{aligned}
& V_{p} \propto \sin (2 \pi \alpha) V\left(2 \omega_{0}\right)+\sin (4 \pi \alpha) V\left(\omega_{0}\right) \\
& V_{e} \propto \sin (2 \pi \alpha) V\left(2 \omega_{0}\right)-\sin (4 \pi \alpha) V\left(\omega_{0}\right),
\end{aligned}
$$

where $V_{p}$ and $V_{e}$ are the effective stripline positron and electron signals that are proportional to the position of only a single species of particle. The stripline voltages defined in Eqs. (12) and (13) are used to perform a difference over sum calculation for opposite BPM striplines to obtain the transverse position of the positrons and electrons that make up the beam.

\section{PROPOSED ELECTRONICS DESIGN TO DETECT THE FUNDAMENTAL AND FIRST HARMONIC STRIPLINE SIGNALS}

Figure 2 shows a block diagram of a prototype electronics system including component specifications that will be used to detect the fundamental ( 2856 $\mathrm{MHz}$ ) and first harmonic ( $5712 \mathrm{MHz})$ BPM stripline signals. The system must extract these components in addition to detecting the phase shift of the first harmonic signal. The front-end electronics separates and filters the fundamental and first harmonic signals using standard components. The 4:1 rf switch selects BPM striplines for signal analysis at the $60-\mathrm{Hz}$ macropulse repetition rate of the linac beam. 


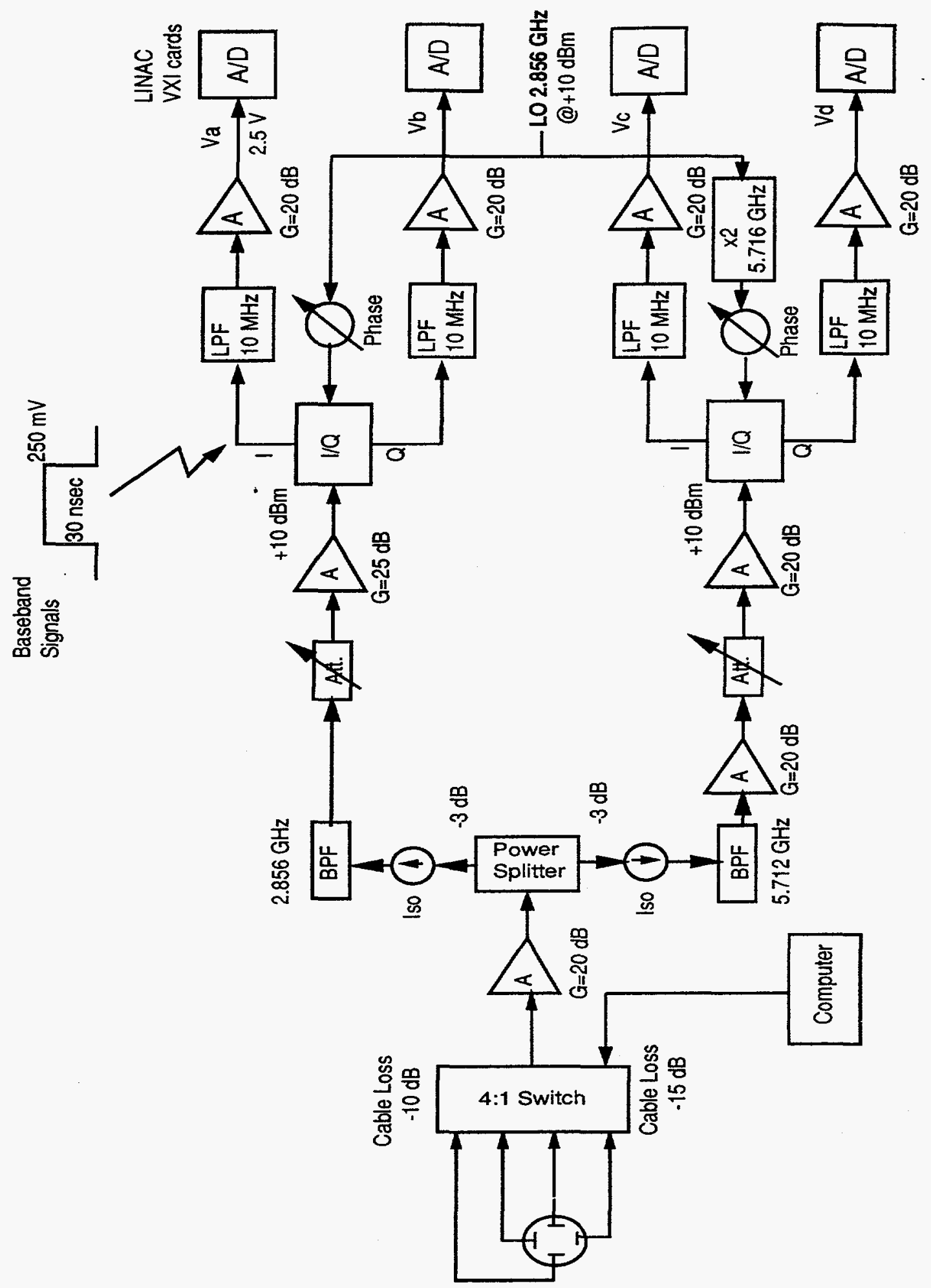

Figure 2. Layout of the prototype circuit used to detect fundamental and first harmonic BPM stripline signals. 
The $1 / Q$ detectors are the basic component used to detect both amplitude and phase of the fundamental and first harmonic signals. The I and Q channels of the detector produce baseband signals proportional to the product of the amplitude of the input signal and the sine and cosine, respectively, of the phase difference between the reference and the input signal. For the first harmonic signal, if the last term on the right-hand side of Eq. (11) changes sign from one linac configuration to the next (either due to current changes, actual beam position changes, or some combination of both), both outputs of the I and Q detector will also change sign. The sum of the squares of the I and $Q$ channels yields the harmonic signal amplitude (squared) and their ratio yields the phase angle between reference and input signal. The I/Q detector thus provides enough information to get each harmonic amplitude and their relative phase with respect to each other. The fundamental and first harmonic signals will be determined in software using the outputs of the I/Q detectors.

Calibration of the system is performed using a pure electron beam which is obtained by simply removing the target. The beam is first centered in the BPM so that the stripline voltages are all equal. The gain of each signal channel is adjusted using variable attenuators so that both fundamental and first harmonic signals have the same amplitude at the entrance to the $\mathrm{A} / \mathrm{D}$ converters. This adjustment effectively takes into account the geometry factor present in the formula for the fundamental and first harmonic signals. The linear combinations given by Eqs. (12) and (13) as well as the final difference over sum position calculation will be performed by software.

\section{Conclusion}

A method of determining the position of a beam containing both positrons and electrons has been presented. It is based on the detection of both fundamental and first harmonic beam signals. A prototype detection circuit was presented based on $\mathrm{I} / \mathrm{Q}$ detectors that will be tested with the standard quarterwavelength stripline BP.Ms installed in the APS linac. Based on these tests, further tests would be made for a one-eighth-wavelength stripline optimized to maximize the first harmonic beam signal since this signal is attenuated by $6 \mathrm{~dB}$ due to the present quarter-wavelength stripline geometry.

\section{Acknowlegements}

The authors would like to acknowledge $W$. Sellyey who proposed a similar idea to detect beam position of a mixed species beam. In addition, M. Borland, G. Decker, E. Kahana, J. Galayda, A. Lumpkin, and M. White provided valuable insight and comments regarding the ideas and analysis presented in this work. 


\section{REFERENCES}

1. M. White, et al., "Performance of the Advanced Photon Source (APS) Linear Accelerator," in Proceedings of the 1995 Particle Accelerator Conference, Dallas, TX, pp. 1073-1075, (1996).

2. R. E. Shafer, AIP Conference Proceedings, 249, 618 (1992).

3. R. E. Shafer, AIP Conference Proceedings, 249, 608 (1992).

4. R. J. Mayhan, Discrete-Time and Continuous-Time Linear Systems, Addison-Wesley Publishing Company, p. 433 (1984).

\section{DISCLAIMER}

This report was prepared as an account of work sponsored by an agency of the United States Government. Neither the United States Government nor any agency thereof, nor any of their employees, makes any warranty, express or implied, or assumes any legal liability or responsibility for the accuracy, completeness, or usefulness of any information, apparatus, product, or process disclosed, or represents that its use would not infringe privately owned rights. Reference herein to any specific commercial product; process, or service by trade name, trademark, manufacturer, or otherwise does not necessarily constitute or imply its endorsement, recommendation, or favoring by the United States Government or any agency thereof. The views and opinions of authors expressed herein do not necessarily state or reflect those of the United States Government or any agency thereof. 